\title{
Branched-chain fatty acid content of foods and estimated intake in the USA
}

\author{
Rinat Rivka Ran-Ressler*†, SangEun Bae, Peter Lawrence, Dong Hao Wang and J. Thomas Brenna* \\ Division of Nutritional Sciences, Cornell University, Ithaca, NY 14853, USA
}

(Submitted 31 January 2014 - Final revision received 20 March 2014 - Accepted 7 April 2014 - First published online 15 May 2014)

\section{Abstract}

Branched-chain fatty acids (BCFA) are bioactive food components that constitute about $2 \%$ of fatty acids in cows' milk fat. There are few systematic data available on the BCFA content of other foods to estimate dietary intakes. In the present study, we report BCFA distribution and content of fresh and processed foods representing the major foods in the American diet and estimate BCFA intake. BCFA are primarily components of dairy and ruminant food products, and are absent from chicken, pork and salmon. The mean BCFA intake of $500 \mathrm{mg} / \mathrm{d}$ was delivered primarily from dairy and beef food products; by comparison, average intake of the widely studied long-chain PUFA EPA and DHA has been estimated to be $100 \mathrm{mg} / \mathrm{d}$. Common adjustments in the diet could double the daily intake of BCFA. The fermented foods sauerkraut and miso had appreciable fractions of BCFA, but, overall, they are low-fat foods providing very small amounts of BCFA in the diet, and other fermented foods did not contain BCFA as might have been expected from the influence of microbial exposure. These data support the quantitative importance of BCFA delivered primarily from dairy and beef food products and highlight the need for research into their effects on health.

\section{Key words: Branched-chain fatty acids: Monomethyl branching: Food composition}

Branched-chain fatty acids (BCFA) are primarily SFA with one methyl branch or more on the carbon chain. BCFA are categorised as mono-, di- or multi-methyl BCFA. In monomethyl BCFA, the predominant branching is near the terminal end of the carbon chain. Fatty acids (FA) terminating with an isopropyl or isobutyl group are referred to as iso- or anteisoBCFA, respectively (Fig. 1). BCFA modulate the biophysical properties of membranes in a manner similar to that of cis double bonds: both types of BCFA interfere with the ability of SFA to pack tightly to form rigid, high-melting point extended structures, and thus reduce the phase transition temperature of membrane phospholipids ${ }^{(1)}$.

BCFA are major components of bacterial membranes across many genera and species. They are particularly prominent in Bacillus, constituting $95 \%$ of the FA in many species of Bacillus and Lactobacillus ${ }^{(1)}$. In some Bifidobacterium strains, a single BCFA concentration can be as high as $24 \mathrm{wt} \%{ }^{(2)}$. When BCFA concentrations are present in large amounts in bacteria, they may influence the phenotype in potentially important ways. For instance, when they are present in the environment, BCFA are readily taken up by the pathogen Pseudomonas aeruginosa and dramatically reduce motility and virulence ${ }^{(3)}$.

BCFA are rare in internal human tissues; however, they are present in high concentrations in the skin and vernix caseosa, the unique waxy white substance coating the skin of term newborns, with their concentrations being about $29 \mathrm{wt} \%{ }^{(4)}$, including BCFA with branches in positions other than the iso and anteiso positions, and also dimethyl BCFA. We have reported previously ${ }^{(4)}$ that BCFA are constituents of the healthy term newborn infant's gut and that the human alimentary canal selectively metabolises BCFA, suggesting that they play a specific role in the gut. Consistent with this hypothesis, our recent study in neonatal rats showed that substitution of $20 \mathrm{wt} \%$ of fat as BCFA altered the gastrointestinal microbial ecology towards organisms that use BCFA and reduced the incidence of necrotising enterocolitis ${ }^{(5)}$. Others $^{(6,7)}$ have shown that BCFA induce apoptosis in human breast cancer cells, and inhibit tumour growth in cultured cells and in a mouse model. These data all point to previously neglected nutritional properties of BCFA that may be important for the development and maintenance of the microbiota, enterocyte health, skin and possibly other functions.

There are scant data on the intake of BCFA in the human diet. We have recently reported the profile and concentrations of BCFA in a representative sampling of retail milk in the USA. Using these data along with the reports of others for BCFA consumption from beef, we estimated the intake of BCFA from cheese and beef food products in the American diet to be about $400 \mathrm{mg} / \mathrm{d}$. However, these calculations were based on retail milk and did not take into account the changes in

Abbreviations: BCFA, branched-chain fatty acids; FA, fatty acids; FAME, fatty acid methyl esters.

*Corresponding authors: R. R. Ran-Ressler, email rrr28@cornell.edu; J. Thomas Brenna, email jtb4@cornell.edu

†Present address: Nestlé R\&D Center Minneapolis, 12500 Whitewater Drive, Minnetonka, MN 55343, USA. 


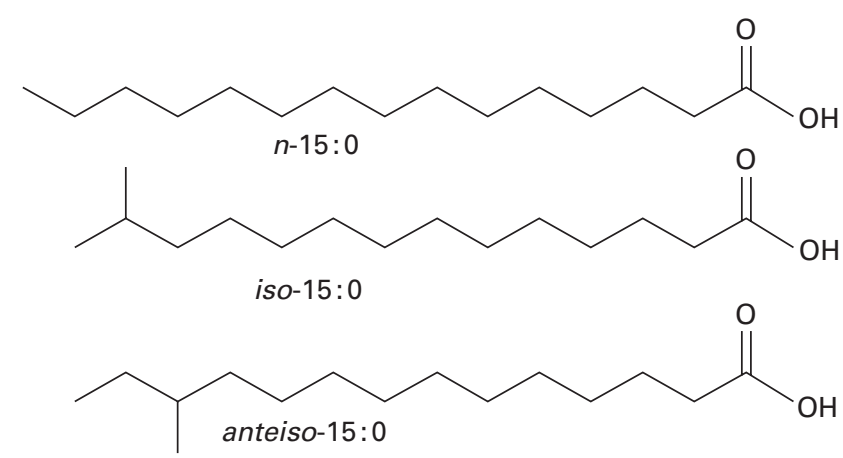

Fig. 1. Structures and naming of representative branched-chain fatty acids (BCFA). $n$ (normal)-Fatty acids have no branching. iso-BCFA have a methyl branch on the penultimate carbon and anteiso-BCFA have a methyl branch on the antepenultimate carbon.

BCFA profiles or the increase or loss in total BCFA concentrations due to, for instance, fermentation or processing methods $^{(8)}$. In the present study, we present the analysis of BCFA in foods prominently featured in the American diet, and estimate from the measurements the nutritional contribution of BCFA from these various foods in the American diet using the USDA's Economic Research Service intake data.

\section{Experimental procedures}

\section{Sampling}

Food samples were purchased from local supermarkets in Ithaca, NY, USA. Because previous reports of BCFA content focus on dairy and meat products, we chose a cross-section from these food groups and additionally included fermented foods. Particular foods were chosen based on the consideration of the prevalence of consumption within respective food groups according to the USDA's Economic Research Service, Loss-Adjusted Food Availability data ${ }^{(9)}$.

The following food products from the dairy food group were analysed: whole milk cheese (bovine milk: cheddar cheese, low-moisture mozzarella cheese, provolone cheese, Swiss cheese, cottage cheese, ricotta cheese, cream cheese; two samples of ovine (sheep) milk-derived cheese (blue, romano); one sample of goat-derived cheese); plain and Greek yogurt; ice cream. We also analysed dairy-based food products from the added fat food group such as sour cream, light cream and butter. However, these foods were merged with the dairy food group to simplify the data analysis.

Foods were chosen based on the prevalence of consumption within the dairy and protein food groups according to the USDA's Economic Research Service, Loss-Adjusted Food Availability data ${ }^{(9)}$. From the protein food group, we analysed ground beef, ground turkey, ham, bacon, pork sausages, chicken thighs, chicken breasts, eggs and almonds. In addition to commercial ground beef, ground beef from a private small farm in northern Pennsylvania, in which beef cattle are exclusively pastured, was also included in the analysis. The most consumed cuts of beef and pork were chosen for analysis ${ }^{(10)}$. Almonds, canned tuna and fresh salmon were also analysed as part of the protein food group.
Fermented food products such as sauerkraut and miso (soya), tofu, kimchi and tempeh were included in the analysis because the addition of bacteria/fungi during the fermentation process of these food products may influence the presence of BCFA. Chocolate bars were also chosen to be included in the analysis as a fat and dairy-containing snack. Samples with a high content of moisture such as yogurt were placed in a centrifugal evaporator (Savant SpeedVac; Thermo Fisher Scientific), for at least $24 \mathrm{~h}$ before the analysis. The samples were stored at $-80^{\circ} \mathrm{C}$ until processed.

\section{Fatty acid analysis}

Samples (100-150 mg) from each food were extracted and methylated according to a modified one-step hydrolysis procedure, as described previously ${ }^{(11,12)}$. Heneicosanoic acid (21:0) was used as the internal standard (Sigma Chemical Company Chemical). Fatty acid methyl esters (FAME) were identified and analysed quantitatively, as discussed in detail previously ${ }^{(8)}$. Briefly, a BPX-70 capillary column $(25 \mathrm{~m} \times 0.22 \mathrm{~mm} \times 0.25 \mu \mathrm{m}$; SGE) with the $\mathrm{H}_{2}$ carrier gas was installed in a HP 5890 gas chromatograph with a flame ionisation detector. A FAME mixture of equal weight (68A; Nu-Chek Prep, Inc.) was used to calculate response factors and six BCFA were used as authentic reference standards (iso-14:0, anteiso-15:0, iso16:0, anteiso-17:0, iso-18:0 and iso-20:0; Larodan Fine Chemicals $\mathrm{AB}$ ). Concentrations of all $\mathrm{FA}$ are expressed as $\mathrm{wt} \%$.

The identities of FAME were determined by electron ionisation MS, chemical ionisation and electron ionisationtandem MS, as described previously ${ }^{(13)}$, using a Varian Star 3400 GC coupled to a Varian Saturn 2000 ion trap MS, with their identities being confirmed by GC retention times. For our protocols with GC-flame ionisation detector analysis, we estimated the limit of quantification to be about $0.15 \mathrm{ng}$ FAME per $1 \mu \mathrm{l}$ injection. For the present study, we prepared approximately $6 \mu \mathrm{g}$ FAME mixture per $1 \mu \mathrm{l}$ injection. These concentrations led to the limit of quantification for individual FAME of about $0.003 \%(\mathrm{w} / \mathrm{w})$ of total FAME.

\section{Results and discussion}

\section{Branched-chain fatty acid content in food samples from a US market}

In the USA, per capita consumption of foods contained in the dairy and protein food groups is 216 and $208 \mathrm{~g} / \mathrm{d}^{(9)}$, respectively. Here, we analysed food products from both of those groups representing $160 \mathrm{~g}$ of $216 \mathrm{~g}$ ( $74 \%$ ) of the per capita daily consumption of dairy food products, and $193 \mathrm{~g}$ of $208 \mathrm{~g}$ (92\%) of the per capita daily consumption of protein food products. The present analysis of the dairy-based added fat food category accounted for $10 \mathrm{~g}$ of $14 \mathrm{~g}$ (sour cream, cream cheese and light cream). We present the results for these dairy-based added fat food groups together with the dairy food group.

Tables 1-3 present in detail the data on BCFA distribution in dairy, protein and fermented food products, respectively. BCFA were detected in dairy and beef food products. Small amounts of BCFA were also detected in canned tuna and in the fermented 


\section{.}

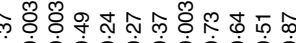

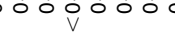

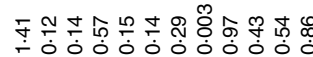

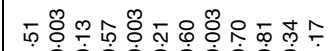

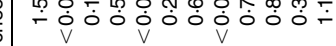

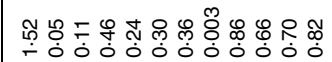

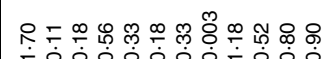

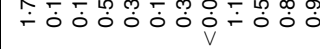

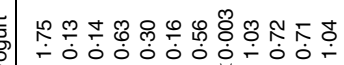
舀

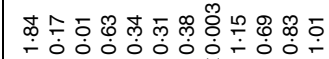

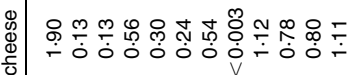

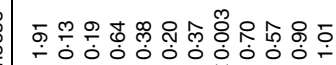

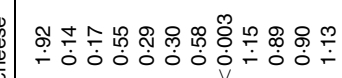

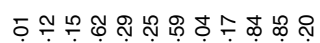

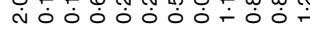

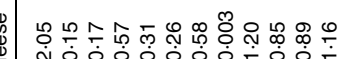

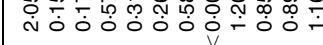

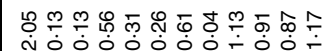

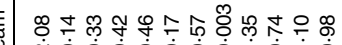

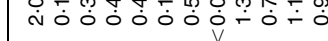

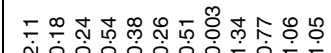

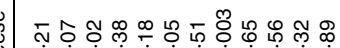

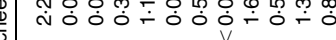

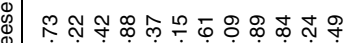

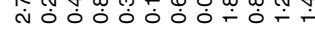

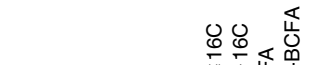

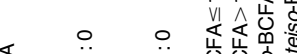

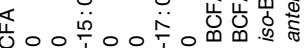

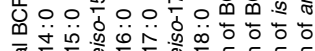

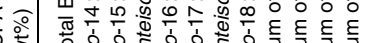

food products miso and sauerkraut. BCFA were found to be below the detection limit in the following food products: ground turkey; ham; bacon; pork sausages; chicken thighs; chicken breasts; eggs; almonds; tofu; tempeh; fresh salmon.

As shown in Table 1, total BCFA concentrations in the various dairy products ranged between $1.37 \mathrm{wt} \%$ for light cream cheese and $2 \cdot 73 \mathrm{wt} \%$ for sheep cheese. Sheep and goat milk have long been known for their higher BCFA concentrations, especially when fed a barley-rich $\operatorname{diet}^{(14)}$. The relatively higher levels of BCFA in sheep cheese and goat cheese show that BCFA concentration in dairy products is influenced by which ruminant species' milk is being used. In addition, differences in cheese composition can be affected by many factors, such as cow breed, feeding practice and microbial activity in milk and cheese, especially during ripening ${ }^{(15)}$. The original milk containing lower concentrations of BCFA that was used in the production of these cows' milk-based food products is the most likely explanation for differences in BCFA content among these dairy products, particularly BCFA concentrations were found to be lower in low-moisture mozzarella cheese than in provolone cheese $(1.4 v .1 .9 \mathrm{wt} \%)$, despite sharing a similar production process.

Fig. 2 shows BCFA distribution in retail cows' milk ${ }^{(8)}$, and in dairy products as a group that derive the bulk of their BCFA from cows' milk. Similar to fluid milk $^{(8)}$, dairy products included both iso and anteiso types of BCFA with chain lengths ranging from 14 to 17 carbons, with very little iso18: 0. In addition, odd-chain anteiso-BCFA comprise at least half of the total BCFA concentrations in cows' milk-based dairy products. The BCFA profile of the present study is in line with BCFA distribution published previously for European dairy products ${ }^{(16,17)}$

Because introduction of bacteria is an integral part of the production of most dairy products and because bacteria can be a source of BCFA - in addition to the inherent presence of BCFA in fluid milk - we speculated that the total BCFA content and distribution may be different between fluid milk and dairy products, with a tendency or trend to be higher in dairy products. Surprisingly, the collective mean levels of total BCFA, anteiso-17:0, the sum of BCFA longer than 16 carbons and the sum of anteiso-BCFA in cows' milk-based dairy products were significantly lower than the mean BCFA levels of these aggregated parameters in retail milk $(P<0 \cdot 05)$. These differences are driven by the higher levels of anteiso-17:0 in retail milk compared with the collective mean levels of BCFA in cows' milk-based dairy products. The cows' diet can strongly affect the levels of FA and BCFA in milk ${ }^{(15)}$, and lower levels of BCFA in milk-based dairy products observed in the present study imply that bacterial BCFA may not have the same prominent effect on BCFA levels in dairy products as do diet and production processes. Furthermore, it is possible that milk BCFA used for the production of some of the dairy products contained somewhat lower levels of BCFA than those in average retail milk, masking possible contributions of bacterial BCFA to the final product. Alternatively, the lack of BCFA in bacterial cultures introduced for the production of dairy food products could explain why BCFA concentrations in cows' milk-based dairy products did not 
Table 2. Total and individual branched-chain fatty acid (BCFA) concentrations in beef and tuna*

\begin{tabular}{lccc}
\hline BCFA (wt\%) & Pastured beef & Commercial beef & Tuna \\
\hline Total BCFA & 1.89 & 1.60 & 0.14 \\
Iso-14:0 & $<0.003$ & $<0.003$ & $<0.003$ \\
Iso-15: 0 & 0.31 & $<0.003$ & $<0.003$ \\
Anteiso-15:0 & 0.24 & 0.21 & $<0.003$ \\
Iso-16:0 & 0.25 & 0.30 & $<0.003$ \\
Iso-17:0 & 0.41 & 0.52 & 0.14 \\
Anteiso-17:0 & 0.51 & 0.57 & $<0.003$ \\
Iso-18:0 & 0.18 & $<0.003$ & $<0.003$ \\
Sum of BCFA $\leq 16 C$ & 0.79 & 0.51 & 0.00 \\
Sum of BCFA $16 C$ & 0.92 & 1.09 & 0.14 \\
Sum of iso-BCFA & 0.96 & 0.82 & 0.14 \\
Sum of anteiso-BCFA & 0.74 & 0.78 & 0.00 \\
\hline
\end{tabular}

* Other foods in the protein food group tested were ground turkey, chicken breasts, chicken thighs, pork sausages, ham, bacon, fresh salmon, eggs and almonds; al had $<0.003$ wt $\%$ BCFA.

increase when compared with those in fluid milk. A literature search for FA composition in known dairy cultures, such as Streptococcus thermophilus and Lactobacillus bulgaricus, did not yield reports on BCFA concentrations in these strains ${ }^{(2,18-20)}$, with the exception of one report on the presence of $0.7 \mathrm{wt} \%$ anteiso-15:0 in Lactobacillus bulgaricus ${ }^{(2)}$. However, the bacterial culture used in making cheese, for example, is important for production of the texture and flavour expected of various cheeses ${ }^{(21)}$, and thus it is reasonable to assume that different dairy products have different mixtures of bacterial cultures. These cultures can have both BCFA-containing and non-BCFA-containing bacterial strains, and the proportions between them may affect the bacterial contribution of BCFA to different dairy products.

In addition to dairy products, we also analysed the most highly consumed foods from the protein food group according to the USDA's Economic Research Service, Loss-Adjusted Food Availability data ${ }^{(9)}$. Table 2 shows the concentrations of BCFA in food products from the protein food group. Commercial and pastured ground beef had total BCFA concentrations of 1.60 and $1.89 \mathrm{wt} \%$. In a recent survey of retail beef in Canada ${ }^{(22)}$, BCFA concentrations ranged between 1.25 and $1.82 \mathrm{wt} \%$ depending on the season and the cut, which is consistent with the present results. Similar to cows' milk, BCFA concentrations in beef can be affected by the cattle's diet, the breed ${ }^{(23,24)}$ and the cut of the meat within a given breed $^{(22)}$. Finally, we noted that production practice such as organic $v$. conventional dairy products, and wild $v$. farmed seafood, may all have an influence on the levels of BCFA.

BCFA concentrations were below the detection limit in poultry and pork products, as would be expected if the primary origin of BCFA in meats is from ruminal bacteria. These observations are in line with a previous report on FA concentrations in lard, chicken fat, mutton tallow and beef tallow, using a GC $\times$ GCtime of flight mass spectrometer ${ }^{(25)}$. A wide variety of BCFA were detected in beef and mutton tallow but not in chicken fat, and a small amount of anteiso-17:0 was reported in lard.

Previous reports have indicated the presence of small amounts of BCFA in fish ${ }^{(17)}$. Because the current 2010 Dietary Guidelines for Americans recommend consumption of two servings of $114 \mathrm{~g}$ ( $4 \mathrm{oz}$ ) each of seafood per week, we investigated the presence of BCFA in canned tuna and salmon, which, among Americans, are the second- and third-most consumed types of seafood (after shrimp), according to the National Oceanic and Atmospheric Administration statistics ${ }^{(26)}$. In addition, these fish are considered to be coldwater fish. Because BCFA modulate membrane properties at low temperatures ${ }^{(1,27)}$, we speculated that BCFA may be present in their membranes. The present data show that BCFA levels in salmon were below the detection limit, and very small amounts of iso-17:0 $(0 \cdot 14 \mathrm{wt} \%)$ were detected in canned tuna. Low concentrations of BCFA in fresh tuna and salmon have been reported by others $^{(17)}$, and the observation of $i s o$-BCFA in canned tuna is in agreement with that of the same authors suggesting that the dominant BCFA in fish are odd-numbered carbon iso-BCFA.

Taken together, the aforementioned results indicate that BCFA are found in high concentrations in ruminant products, but not in poultry products and at zero or low amounts in the fish that we examined. Considering that the foods in the present study were the most commonly consumed foods in the US diet, the present data indicate that the main source of BCFA in the dairy and protein food groups originates from ruminant products. In addition, BCFA levels in cows' milk-based dairy products were similar to or lower than those detected in retail cows' milk.

BCFA levels were also detected in fermented products such as sauerkraut and miso (Table 3 ). These products undergo fermentation with various fungi and bacteria, both of which may serve as a source of BCFA in these products. Miso is processed by using the fungus Aspergillus oryzae, a variety of bacterial strains such as Enterococcus and Pediococcus, as well as Lactobacillus and Weissella ${ }^{(28)}$. In the present study, total BCFA concentrations in miso were found to be $0.54 \mathrm{wt} \%$. These included a small amount of iso-15:0 (0.08 wt \%); the rest $(0.46 \mathrm{wt} \%$; Table 3) was contributed by a unique BCFA with a putative assignment of 2-methyl hexadecanoic acid. This rare BCFA was also present at a lower concentration in sauerkraut (0.14 wt $\%$; Table 3). Park et al. ${ }^{(29)}$ reported the presence of Weissella in sauerkraut, which may explain the presence

Table 3. Total and individual branched-chain fatty acid (BCFA) concentrations in sauerkraut and miso*

\begin{tabular}{lcc}
\hline BCFA (wt\%) & Sauerkraut & Miso \\
\hline Total BCFA & 1.02 & 0.54 \\
Iso-14:0 & $<0.003$ & $<0.003$ \\
Iso-15:0 & $<0.003$ & 0.08 \\
Anteiso-15:0 & $<0.003$ & $<0.003$ \\
Iso-16:0 & 0.23 & $<0.003$ \\
2-Methyl-16:0 & 0.14 & 0.46 \\
Iso-17:0 & $<0.003$ & $<0.003$ \\
Anteiso-17:0 & 0.35 & $<0.003$ \\
Iso-18:0 & 0.31 & $<0.003$ \\
Sum of BCFA $\leq 16 C$ & 0.23 & 0.08 \\
Sum of BCFA $>16 C$ & 0.80 & 0.46 \\
Sum of iso-BCFA & 0.54 & 0.08 \\
Sum of anteiso-BCFA & 0.35 & 0.00 \\
\hline
\end{tabular}

* Other fermented foods tested were tofu, tempeh and kimchi; all had $<0.003$ wt $\%$ BCFA. 


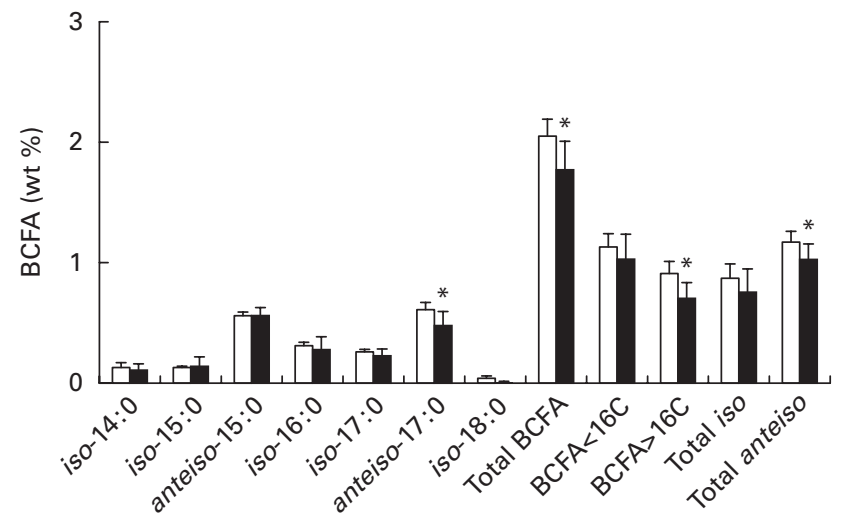

Fig. 2. Description of individual branched-chain fatty acids (BCFA), total BCFA and chain-length distribution of BCFA in fluid milk $(\square) v$. dairy products $(\square)$. Fluid milk data were obtained from our previous publication ${ }^{(8)}$. Values are means, with their standard deviations represented by vertical bars. * Mean values were significantly lower than those of the aggregated parameters in retail milk $(P<0.05)$.

of this BCFA in sauerkraut and miso. A study on FA distribution in Weissella strains did not report $\mathrm{BCFA}^{(30)}$.

BCFA were, however, not detected in other fermented food products such as kimchi or tempeh. Kimchi, for example, contains many different bacterial genera ${ }^{(31)}$, and thus it is likely that BCFA present in some strains were diluted to below the detection limit by the overwhelming amounts of bacteria with normal FA. In addition, various processing methods may also influence bacterial ecology in kimchi ${ }^{(31)}$ and the detection of BCFA in other fermented food products. However, we did not attempt here to do an exhaustive sampling of fermented foods.

Small amounts of BCFA $(0 \cdot 15 \mathrm{wt} \%)$ were detected in milk chocolate. The two BCFA detected in chocolate had 17 carbons, iso-17:0 (0.07 wt \%) and anteiso-17:0 (0.08 wt \%) (data not shown). The ratio between $i s o-17: 0$ and anteiso- $17: 0$ in chocolate (1:1) is different from their ratio in milk (1:2 or more). This and the absence of anteiso-15:0, a predominant BCFA in milk, implies that the milk ingredient in chocolate is not solely responsible for the presence of BCFA in chocolate, and there may be another source of these two BCFA in chocolate.

\section{Branched-chain fatty acid intake of Americans compared with consumption of other bioactive fatty acids}

In the present study, the contribution of BCFA to the nutrition of Americans was estimated from measured and estimated intakes of the dairy and protein food products. The intake data were estimated using the USDA's Economic Research Service, Loss-Adjusted Food Availability data ${ }^{(9)}$. The nutritional contribution of BCFA from various dairy and protein food groups analysed in the present study and their data on a per capita consumption basis that is greater than $1 \mathrm{~g} / \mathrm{d}$ are given in Table 4 . The contribution of the selected foods, presented in Table 4 , to the consumption of BCFA by the US population reaches to a level of about $492 \mathrm{mg} /$ capita per d. Americans consume about $317 \mathrm{mg}$ BCFA from dairy products and about

Table 4. Estimated per capita intake of branched-chain fatty acids (BCFA) from the dairy and protein food groups in the US population

\begin{tabular}{|c|c|c|c|c|c|}
\hline Food & Food consumed $(\mathrm{g} / \mathrm{d})^{*}$ & Fat $(\%)^{*}$ & Fat consumed $(\mathrm{g} / \mathrm{d})$ & BCFA (\%) & BCFA consumed $(\mathrm{mg} / \mathrm{d})$ \\
\hline Beef (cooked) & 53.9 & $18 \cdot 0$ & $9 \cdot 7$ & $1.8 \dagger$ & 169.8 \\
\hline Cheddar cheese & $13 \cdot 7$ & 33.0 & 4.5 & $1 \cdot 7$ & $76 \cdot 9$ \\
\hline Butter & 3.7 & $80 \cdot 0$ & 3.0 & 1.7 & 54.4 \\
\hline Mozzarella cheese & $9 \cdot 1$ & $22 \cdot 1$ & $2 \cdot 0$ & 1.4 & $28 \cdot 3$ \\
\hline Plain whole milk & $40 \cdot 5$ & $3 \cdot 3$ & 1.3 & $2 \cdot 0$ & $26 \cdot 3$ \\
\hline Ice cream & $11 \cdot 0$ & $11 \cdot 0$ & $1 \cdot 2$ & $2 \cdot 1$ & 25.4 \\
\hline $2 \%$ Milk & $52 \cdot 7$ & $2 \cdot 0$ & $1 \cdot 1$ & $2 \cdot 0$ & $21 \cdot 1$ \\
\hline Light cream & $7 \cdot 2$ & $19 \cdot 3$ & 1.4 & 1.4 & $19 \cdot 1$ \\
\hline American cheese & 2.9 & $25 \cdot 6$ & 0.7 & 1.9 & $14 \cdot 2$ \\
\hline Sour cream & $4 \cdot 1$ & $19 \cdot 8$ & 0.8 & 1.5 & $12 \cdot 3$ \\
\hline Cream cheese & $2 \cdot 3$ & 34.0 & 0.8 & 1.5 & $11 \cdot 8$ \\
\hline Yogurt & $11 \cdot 7$ & $3 \cdot 3$ & 0.4 & $1.9 \dagger$ & $7 \cdot 2$ \\
\hline Provolone cheese & $1 \cdot 2$ & $26 \cdot 4$ & 0.3 & 1.9 & 5.9 \\
\hline $1 \%$ Milk & $20 \cdot 6$ & $1 \cdot 0$ & 0.2 & $2 \cdot 0$ & $4 \cdot 1$ \\
\hline Swiss cheese & 0.7 & $27 \cdot 6$ & 0.2 & $2 \cdot 0$ & $4 \cdot 1$ \\
\hline Sheep cheeseł & 0.3 & $31 \cdot 0$ & 0.1 & $2 \cdot 7$ & 2.5 \\
\hline Ricotta cheese & 0.9 & $13 \cdot 0$ & $0 \cdot 1$ & 1.9 & $2 \cdot 2$ \\
\hline Blue cheese (sheep) & 0.1 & 28.5 & 0.0 & $2 \cdot 1$ & 0.7 \\
\hline Cottage cheese & $0 \cdot 8$ & $4 \cdot 3$ & 0.0 & $1 \cdot 8$ & $0 \cdot 6$ \\
\hline Tuna (canned) & $2 \cdot 6$ & $4.6 \dagger$ & 0.1 & 0.1 & 0.2 \\
\hline Chocolate§ & $12 \cdot 6$ & $30 \cdot 0$ & $3 \cdot 8$ & 0.1 & $5 \cdot 7$ \\
\hline Total BCFA & & & & & 492 \\
\hline
\end{tabular}

*Data on food consumed are obtained from the USDA ${ }^{(9)}$, and data on fat content taken from the USDA National Nutrient Database for Standard Reference, Release 25 (http://www.ars.usda.gov/Services/docs.htm?docid=8964), and USDA-ARS 2012. Nutrient Intakes from Food: Mean Amounts Consumed per Individual, by Gender and Age, What We Eat in America, NHANES 2009-2010. Available at www.ars.usda.gov/ba/bhnrc/fsrg. Both databases accessed December 2012.

†For estimation of intake, the following were calculated and used: beef - mean BCFA levels in retail and small-farm pastured beef; yogurt - mean BCFA in two major styles (plain yogurt and Greek yogurt); tuna - mean fat concentrations in tuna canned in oil and tuna canned in water. $\ddagger$ Per capita consumption data were obtained from the National Research Council ${ }^{(43)}$

$\S$ Per capita consumption data in the USA were obtained from Seligson et al. ${ }^{(44)}$. 


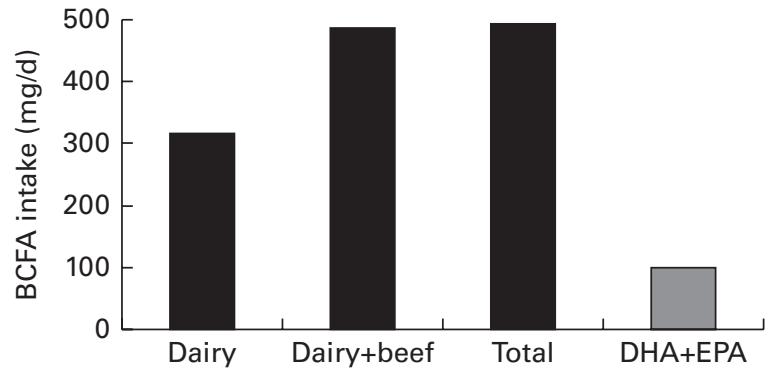

Fig. 3. Daily branched-chain fatty acid (BCFA) intake $v$. intake of bioactive fatty acids DHA and EPA, calculated from the US Department of Agriculture's intake data. See text for details.

$170 \mathrm{mg}$ BCFA from beef. Consumption of chocolate contributes about $6 \mathrm{mg} \mathrm{BCFA} / \mathrm{d}$. Concentrations of BCFA were estimated to be 0.6 and $1.9 \mathrm{wt} \%$ from the total daily intake of $76.8 \mathrm{~g}$ fat and $25.5 \mathrm{~g}$ saturated fat, respectively, for American children aged 2 years and older.

Notable examples of foods excluded from Table 4 are as follows: goat cheese, which contains relatively large amounts of fat $(27 \%)$ and BCFA $(2 \cdot 2 \mathrm{wt} \%)$ but has no per capita consumption data; miso, whose per capita consumption is not tabulated; sauerkraut, whose per capita consumption is very small $(<0 \cdot 4 \mathrm{~g} / \mathrm{d})$ and fat contribution is negligible.

The estimated per capita BCFA intake of $492 \mathrm{mg} / \mathrm{d}$ from the dairy and protein food groups is greater than the average DHA and EPA consumption of $100 \mathrm{mg} / \mathrm{d}$ reported in a survey of 8604 Americans between 1999 and 2000 and in women of childbearing age, based on NHANES III data ${ }^{(32,33)}$. The estimated per capita intake of BCFA is higher than the recommended intake of DHA and EPA for pregnant and lactating women ${ }^{(33)}$. Therefore, the consumption of BCFA from the dairy and protein food products is almost five times the consumption of DHA and EPA in the US population (Fig. 3). As a reference, DHA intake of $200 \mathrm{mg} / \mathrm{d}$, and $300 \mathrm{mg} \mathrm{DHA}+\mathrm{EPA}$ (combined), is recommended by the FAO and WHO for pregnant and lactating women to support infant development ${ }^{(34)}$.

For children aged 2-11 years, the mean intake of beef is $43.5 \mathrm{~g} / \mathrm{d}^{(35)}$. Children who consume $28 \mathrm{~g}$ ( $\left.1 \mathrm{oz}\right)$ of cows' milk-based cheese with an average of $25 \%$ fat, and $43.5 \mathrm{~g} / \mathrm{d}$ of cooked beef with an average of $18 \%$ fat, both with an average BCFA concentration of $1.8 \mathrm{wt} \%$, consume about $267 \mathrm{mg}$ $\mathrm{BCFA} / \mathrm{d}$. The addition of one cup of whole milk $(156 \mathrm{mg}$ BCFA) increases their BCFA intake to $423 \mathrm{mg} / \mathrm{d}$. For comparison, the mean intakes of DHA and arachidonic acid in Canadian children, aged 4-7 years, have recently been estimated to be 37 and $57 \mathrm{mg} / \mathrm{d}^{(36)}$; both DHA and arachidonic acid combined to provide about one-fifth of the daily BCFA consumption from milk, cheese and beef.

Taken together, the aforementioned calculations imply that BCFA are being consumed in substantial amounts by most non-vegans, during different life stages, and their consumption exceeds the consumption of bioactive FA.

The present data indicate that intake of BCFA can exceed the estimated per capita consumption of $492 \mathrm{mg} / \mathrm{d}$ by at least 2 -fold with common intakes of popular foods. For example, daily consumption of $150 \mathrm{~g}(5 \cdot 3 \mathrm{oz})$ of cooked ground beef
(18\% fat, with $1.8 \mathrm{wt} \%$ BCFA on average), two cups ( $473 \mathrm{ml})$ of whole milk $(3.25 \%$ fat, $2 \mathrm{wt} \%$ BCFA) and $57 \mathrm{~g}(2 \mathrm{oz})$ of cows' milk-based cheese ( $25 \%$ fat, $1.8 \mathrm{wt} \%$ BCFA on average) contains about $1050 \mathrm{mg}$ BCFA. If cows' milk-based cheese was replaced with sheep cheese ( $31 \%$ fat and $2.7 \mathrm{wt} \% \mathrm{BCFA}$ ), then meal BCFA concentration would exceed $1700 \mathrm{mg}$ or 3 -fold the average intake.

The 2010 Dietary Guidelines for Americans ${ }^{(37)}$ recommend that Americans consume low-fat ruminant products in order to lower the consumption of saturated fat and to reduce the risk of metabolic diseases. The present results indicate that a higher intake of BCFA is due to the consumption of animal fat and more specifically to the intake of foods of ruminant origin, with the leading foods in the American diet being dairy and beef food products. A recent meta-analysis of prospective cohort studies has failed to find a significant relationship between dietary saturated fat intake and the risk of $\mathrm{CVD}^{(38)}$. Others ${ }^{(39)}$ have shown that increased consumption of high-fat dairy products did not significantly increase the OR for obesity, abnormal glucose homeostasis, dyslipidaemia, elevated blood pressure or insulin resistance. In fact, the OR for these disorders were found to be lower with increasing intake of both reducedfat and high-fat dairy products, and inverse associations were observed for both reduced-fat and high-fat dairy products. Another study has reported a non-significant trend towards lower risk for first myocardial infarction with consumption of high-fat dairy products compared with consumption of lowfat dairy products ${ }^{(40)}$. Another meta-analysis has found no association between animal fat intake and the risk of colorectal cancer $^{(41)}$. Others ${ }^{(42)}$ have shown that intake of saturated fat from an animal source was not significantly associated with the risk of colorectal cancer in women; in that study, consumption of red meat was found to be negatively associated with the risk of colorectal cancer. These studies highlight evolving science on saturated fat intake and disease risks, indicating that the effect of saturated fat or animal fat consumption on metabolic diseases and cancer is complex.

BCFA are normal constituents present in the gut from a very early age and throughout the human life cycle. Previous studies have shown that BCFA are not inert components of the gastrointestinal tract and are metabolised by the enterocytes. BCFA play a beneficial role against inflammation in the premature intestine, alter the microbiota and increase the expression of anti-inflammatory cytokines in an animal model. These studies have shown that BCFA may have a beneficial effect on proper gut functions; thus, their intake in the population becomes relevant. The present study was the first to investigate a wide range of food products consumed by the US population. The present data show that the per capita daily consumption of BCFA is substantial and above the consumption of bioactive $n-3$ FA such as EPA and DHA. The prominence of BCFA in the US food supply and their bioactivity strongly suggest that the effects of BCFA on health should be studied.

\section{Acknowledgements}

The present study was supported by NIH grants T32 HD007331 and R21 HD064604. 


\section{References}

1. Kaneda T (1991) Iso- and anteiso-fatty acids in bacteria: biosynthesis, function, and taxonomic significance. Microbiol Rev 55, 288-302.

2. Veerkamp JH (1971) Fatty acid composition of Bifidobacterium and Lactobacillus strains. J Bacteriol 108, 861-867.

3. Inoue T, Shingaki R \& Fukui K (2008) Inhibition of swarming motility of Pseudomonas aeruginosa by branched-chain fatty acids. FEMS Microbiol Lett 281, 81-86.

4. Ran-Ressler RR, Devapatla S, Lawrence P, et al. (2008) Branched chain fatty acids are constituents of the normal healthy newborn gastrointestinal tract. Pediatr Res 64, 605-609.

5. Ran-Ressler RR, Khailova L, Arganbright KM, et al. (2011) Branched chain fatty acids reduce the incidence of necrotizing enterocolitis and alter gastrointestinal microbial ecology in a neonatal rat model. PLOS ONE 6, e29032.

6. Yang Z, Liu S, Chen X, et al. (2000) Induction of apoptotic cell death and in vivo growth inhibition of human cancer cells by a saturated branched-chain fatty acid, 13-methyltetradecanoic acid. Cancer Res 60, 505-509.

7. Wongtangtintharn $\mathrm{S}$, Oku H, Iwasaki $\mathrm{H}$, et al. (2004) Effect of branched-chain fatty acids on fatty acid biosynthesis of human breast cancer cells. J Nutr Sci Vitaminol (Tokyo) 50, 137-143

8. Ran-Ressler RR, Sim D, O'Donnell-Megaro AM, et al. (2011) Branched chain fatty acid content of United States retail cow's milk and implications for dietary intake. Lipids $\mathbf{4 6}$, 569-576.

9. USDA (2012) Loss-Adjusted Food Availability data. http://www. ers.usda.gov/data-products/food-availability-(per-capita)data-system.aspx\#26705 (accessed January 2013).

10. Davis CG \& Lin BH (2005) Factors Affecting U.S. Beef Consumption. Washington, DC: USDA

11. Garces R \& Mancha M (1993) One-step lipid extraction and fatty acid methyl esters preparation from fresh plant tissues. Anal Biochem 211, 139-143.

12. Zhou Y, Nijland M, Miller M, et al. (2008) The influence of maternal early to mid-gestation nutrient restriction on long chain polyunsaturated fatty acids in fetal sheep. Lipids $\mathbf{4 3}$, 525-531.

13. Ran-Ressler RR, Lawrence P \& Brenna JT (2012) Structural characterization of saturated branched chain fatty acid methyl esters by collisional dissociation of molecular ions generated by electron ionization. J Lipid Res 53, 195-203.

14. Duncan WR \& Garton GA (1978) Differences in the proportions of branched-chain fatty acids in subcutaneous triacylglycerols of barley-fed ruminants. Br J Nutr 40, 29-33.

15. Bonanno A, Tornambe G, Bellina V, et al. (2013) Effect of farming system and cheesemaking technology on the physicochemical characteristics, fatty acid profile, and sensory properties of Caciocavallo Palermitano cheese. J Dairy Sci 96, 710-724.

16. Thurnhofer S, Lehnert K \& Vetter W (2008) Exclusive quantification of methyl-branched fatty acids and minor 18:1-isomers in foodstuff by GC/MS in the SIM mode using 10,11dichloroundecanoic acid and fatty acid ethyl esters as internal standards. Eur Food Res Technol 226, 975-983.

17. Hauff S \& Vetter W (2010) Quantification of branched chain fatty acids in polar and neutral lipids of cheese and fish samples. J Agric Food Chem 58, 707-712.

18. Smittle RB, Gilliland SE, Speck ML, et al. (1974) Relationship of cellular fatty acid composition to survival of Lactobacillus bulgaricus in liquid $\mathrm{N}_{2}$. Appl Microbiol 27, 738-743.

19. Beal C, Fonseca F \& Corrieu G (2001) Resistance to freezing and frozen storage of Streptococcus thermophilus is related to membrane fatty acid composition. I Dairy Sci $\mathbf{8 4}$ $2347-2356$

20. Tymczyszyn EE, Gomez-Zavaglia A \& Disalvo EA (2005) Influence of the growth at high osmolality on the lipid composition, water permeability and osmotic response of Lactobacillus bulgaricus. Arch Biochem Biophys 443, 66-73.

21. Randazzo CL, De Luca S, Todaro A, et al. (2007) Preliminary characterization of wild lactic acid bacteria and their abilities to produce flavour compounds in ripened model cheese system. J Appl Microbiol 103, 427-435.

22. Aldai N, Dugan MER, Rolland DC, et al. (2009) Survey of the fatty acid composition of Canadian beef: backfat and longissimus lumborum muscle. Can J Anim Sci 89, 315-329.

23. Kraft J, Kramer JK, Schoene F, et al. (2008) Extensive analysis of long-chain polyunsaturated fatty acids, CLA, trans-18:1 isomers, and plasmalogenic lipids in different retail beef types. J Agric Food Chem 56, 4775-4782.

24. Costa AS, Lopes PA, Estevao M, et al. (2012) Contrasting cellularity and fatty acid composition in fat depots from Alentejana and Barrosa bovine breeds fed high and low forage diets. Int J Biol Sci 8, 214-227.

25. Chin ST, Man YBC, Tan CP, et al. (2009) Rapid profiling of animal-derived fatty acids using fast GC $\times$ GC coupled to time-of-flight mass spectrometry. J Am Oil Chem Soc 86, 949-958.

26. NOAA (2013) NOAA Fisheries. http://www.nmfs.noaa.gov/ aquaculture/faqs/faq_seafood_health.html\#6how (accessed March 2013)

27. Annous BA, Becker LA, Bayles DO, et al. (1997) Critical role of anteiso-C15:0 fatty acid in the growth of Listeria monocytogenes at low temperatures. Appl Environ Microbiol $\mathbf{6 3}$, 3887-3894.

28. Onda T, Yanagida F, Tsuji M, et al. (2003) Time series analysis of aerobic bacterial flora during Miso fermentation. Lett Appl Microbiol 37, 162-168.

29. Park EJ, Kim KH, Abell GC, et al. (2011) Metagenomic analysis of the viral communities in fermented foods. Appl Environ Microbiol 77, 1284-1291.

30. Samelis J, Rementzis J, Tsakalidou E, et al. (1998) Usefulness of rapid GC analysis of cellular fatty acids for distinguishing Weissella viridescens, Weissella paramesenteroides, Weissella bellenica and some non-identifiable, arginine-negative Weissella strains of meat origin. Syst Appl Microbiol 21, 260-265.

31. Park EJ, Chun J, Cha CJ, et al. (2012) Bacterial community analysis during fermentation of ten representative kinds of kimchi with barcoded pyrosequencing. Food Microbiol 30, 197-204

32. Ervin RB, Wright JD, Wang CY, et al. (2004) Dietary intake of fats and fatty acids for the United States population: 1999-2000. Adv Data 1-6.

33. Brenna JT \& Lapillonne A (2009) Background paper on fat and fatty acid requirements during pregnancy and lactation. Ann Nutr Metab 55, 97-122.

34. Anonymous (2010) Fats and Fatty Acids in Human Nutrition. Report of an Expert Consultation. FAO Food and Nutrition Paper 91. Rome, Italy: Food and Agriculture Organization of the United Nations.

35. Daniel CR, Cross AJ, Koebnick C, et al. (2011) Trends in meat consumption in the USA. Public Health Nutr 14, 575-583.

36. Lien VW \& Clandinin MT (2009) Dietary assessment of arachidonic acid and docosahexaenoic acid intake in 4-7 year-old children. I Am Coll Nutr 28, 7-15.

37. USDA (2010) Dietary Guidelines for Americans. Washington, DC: USDA.

38. Siri-Tarino PW, Sun Q, Hu FB, et al. (2010) Meta-analysis of prospective cohort studies evaluating the association of 
saturated fat with cardiovascular disease. Am J Clin Nutr $\mathbf{9 1}$, $535-546$

39. Pereira MA, Jacobs DR Jr, Van Horn L, et al. (2002) Dairy consumption, obesity, and the insulin resistance syndrome in young adults: the CARDIA Study. JAMA 287, 2081-2089.

40. Lockheart MS, Steffen LM, Rebnord HM, et al. (2007) Dietary patterns, food groups and myocardial infarction: a casecontrol study. Br J Nutr 98, 380-387.

41. Alexander DD, Cushing CA, Lowe KA, et al. (2009) Metaanalysis of animal fat or animal protein intake and colorectal cancer. Am J Clin Nutr 89, 1402-1409.
42. Lin J, Zhang SM, Cook NR, et al. (2004) Dietary fat and fatty acids and risk of colorectal cancer in women. $A m J$ Epidemiol 160, 1011-1022.

43. National Research Council (2008) Changes in the Sheep Industry in the United States: Making the Transition from Tradition, Chapter 6. Committee on the Economic Committee on the Economic Development and Current Status of the Sheep Industry in the United States, National Research Council. Washington, DC: National Research Council.

44. Seligson FH, Krummel DA \& Apgar JL (1994) Patterns of chocolate consumption. Am J Clin Nutr 60, 1060S-1064S. 